Article

\title{
Threat and Anxiety in the Climate Debate-An Agent-Based Model to Investigate Climate Scepticism and Pro-Environmental Behaviour
}

\author{
Marie Lisa Kapeller *(D) and Georg Jäger *(1) \\ Institute of Systems Sciences, Innovation and Sustainability Research, University of Graz, 8010 Graz, Austria \\ * Correspondence: marie.kapeller@uni-graz.at (M.L.K.); georg.jaeger@uni-graz.at (G.J.)
}

Received: 22 January 2020; Accepted: 24 February 2020; Published: 28 February 2020

\begin{abstract}
In order to meet the challenges of sustainable development, it is of utmost importance to involve all relevant decision makers in this process. These decision makers are diverse, including governments, corporations and private citizens. Since the latter group is the largest and the majority of decisions relevant to the future of the environment is made by that group, great effort has been put into communicating relevant research results to them. The hope is that well-informed citizens make well-informed choices and thus act in a sustainable way. However, this common but drastic simplification that more information about climate change automatically leads to pro-environmental behaviour is fundamentally flawed. It completely neglects the complex social-psychological processes that occur if people are confronted with threatening information. In reality, the defence mechanisms that are activated in such situations can also work against the goal of sustainable development, as experimental studies showed. Based on these findings, we propose an agent-based model to understand the relation between threatening climate change information, anxiety, climate change scepticism, environmental self-identity and pro-environmental behaviour. We find that the exposure to information about climate change, in general, does not increase the pro-environmental intent unless several conditions regarding the individual's values and information density are met.
\end{abstract}

Keywords: agent-based model; social contagion; networks; climate scepticism; pro-environmental behaviour; anxiety; decision

\section{Introduction}

Most research dedicated to mitigating climate change faces a delicate issue: From a research point of view, possible paths that can lead to sustainable development are, at least in general, quite clear. The main changes in our lifestyle that are required in order to limit the damage of climate change, are well understood and there is, for example, consensus that our current form of mobility and reliance on fossil fuels is unsustainable. The realisation of greener lifestyles, however, is proceeding slowly and has been facing stagnation for decades: Per capita consumption of meat continues to increase [1], commercial aviation is the fastest growing source of greenhouse gas (GHG) emissions [2], the carbon footprint of global tourism is increasing faster than anticipated [3], private car usage is on the rise and an overall higher energy consumption in the transport sector [4] are a few of the identified trends in an individual's carbon emissions.

Thus it is clear that in order to reach the goal of coping with the threat of climate change $[5,6]$ it is vital to motivate citizens to adopt more environmentally friendly habits, especially promoting high effort pro-environmental behaviour [7,8]. The usual approach for this, chosen by most environmental organisations, governments and involved stakeholders, is to expose citizens to the risks and dangers of 
climate change. The assumption behind this seems intuitively correct: More awareness of the problem of climate change leads to more climate-friendly actions. However, the assumption that awareness inevitably results in appropriate behavioural change has been challenged before [9,10]. Even more so, threatening climate messages can create unplanned effects and may also backfire [9]. Drawing from social-psychological research, responses to climate change messaging can be complex and include avoiding or negating the problem which can in the end destroy parts of the progress we already made towards more sustainable lifestyles.

Despite the profound scientific findings on climate change, public and media discussions are often distorted and shifted towards a discussion about whether the scientific facts are valid at all [11]. While this shift in discussion bias towards climate change denial is criticised by climate scientists among several voices, it is often unintentionally supported by failing to take sufficient account of the social-psychological reasons that can lead to denial of climate change. For further information on that aspect, see Section 2.

The purpose of this study is to contribute to an understanding of the societal phenomenon of responses to threatening information on climate change. Drawing on social-psychological research, we have developed a computer model that describes the scenario in which negative information is disseminated in a group or population. In this contribution we present an agent-based model to investigate the time evolution of climate scepticism and pro-environmental behaviour of interacting individuals. Our main goal is to evaluate the model dynamics to identify conditions which can support favourable scenarios such as an increase in the intention to act environmentally friendly, and to examine circumstances which enhance or dampen possible backfiring effects such as an increase of the climate scepticism. Our aim is to generate and extend certain hypotheses about so far poorly understood effects of the climate debate. At the same time we would like to point out that this analytical study is a schematic representation of certain phenomena and should not be understood as a political directive.

\section{Threat and Defence Model: Theoretical Background}

The social-psychological threat literature deals with reactions to problems such as personal uncertainty, loss of control, conflicting goals or perceptual surprises. Recently, an 'integrative general model of threat and defence processes' was developed that provides a conceptual framework for understanding such diverse phenomena [12]. Based on societal psychological and neural perspectives on defensive reactions to threat, the model proposes the simple hypothesis that discrepancies arouse anxiety and thereby motivate diverse phenomena to relieve that anxiety. From this perspective, threats result from an experience of a discrepancy between an expectation or a desire and the current circumstances.

This perceived threat is followed by anxiety, which leads to a variety of proximal defence reactions such as avoiding the problem. The threat-related processing is mediated by the Behavioural Inhibition System (BIS), which responds with symptoms such as anxious arousal and avoidance. In case of potentially threatening information, individuals increase their efforts to suppress or distract and distance themselves from identified anxious thoughts or circumstances.

A second way to combat anxiety is to turn to approach-motivated behaviour. This reaction pattern manifests itself through the Behavioural Approach System (BAS). When activated, the possible responses include various strategies to seek an effective solution to the problem at hand. Activation is preferred when the discrepancy appears manageable. Since approach-motivated states are able to dampen anxiety and conflict, the anxious BIS stage is successively supplanted or shortened. In case of no available solutions to the threat (e.g., impending death due to ongoing sickness) individuals can nevertheless turn to approach-motivated states by indirectly solving the threat through soothing or mellowing reactive patterns.

In a nutshell, when people try to relieve anxiety in an active BIS state, they tend to symbolic actions and turn to more rewarding aspects of life, even if these aspects are unrelated to the actual threat at hand or its solution. However, in the course of time, most people eventually 
succeed in muting the BIS. Countering anxiety by activation of the BAS state produces distal approach-oriented reactions, helping to sooth anxiety by regaining stability and overcoming this negative state. The BAS can be empowered by the pursuit of personal goals by providing a target for the approach-motivated behaviour. Personal goals can be derived from internal orientations (self-identity, values) or social norms.

\subsection{An Empirical Study on Climate Change Messaging}

The nature of the threat posed by negative climate information is not an actual physical threat, but a perceived threat in the mind based on neurological processes [12]. After a stimulus, the threat experience serves as an activating alarm cue to which people immediately respond by a variety of reactions. A recent study examined these responses by exposing participants to negative consequences of climate change [9,10] with reference to the threat and defence model [12]. The respective approach-motivated behaviour refers to an increase in the intention to act environmentally friendly. Participants evaluated several climate change statements, regarding whether they are interesting or not. Afterwards their pro-environmental intention was retrieved and compared to a control group. The study measures the pro-environmental behaviour in terms of intentions that encompass different activities spanning from household energy saving, waste production and disposal, environmental protection, buying energy-intensive products, transportation preferences to the willingness to make an additional financial effort to protect the environment.

The main finding of the empirical surveys on climate information responses $[9,10]$ is that when being confronted with climate change impacts, participants with high environmental self-identity, i.e., the extent to which people see themselves as acting environmentally-friendly, exhibit higher intentions to act pro-environmental. At the same time these individuals show symbolic defensive behaviour by looking more negatively at multiple groups, including criminals, overweight, or unattractive people. The current explanation for this is that high environmental identity leads to a greater perceived threat for these participants, who are then unable to fully resolve the anxiety through purely direct behaviour. These studies show a high congruence with similar empirical surveys regarding threat responses $[13,14]$.

\subsection{Threat in the Climate Debate}

Interestingly, when discussing the lack of willingness to adopt environmentally friendly behaviour, it is often assumed that too little knowledge about a subject is the cause of the inability to recognise a problem. Although this may be partially true, there are often psychological barriers that prevent us from reacting appropriately [15] although people who behave in a more pro-environmental way are actually more satisfied with their lives [16]. People generally tend to circumvent threats over which they feel they have no control [12]. Negative reports about future risks of an unstable climate system provide a striking example on this issue. Especially the complexity, scale and multidimensional nature of the system earth and its climate can be intimidating, even more so, frightening when discussing potential hazards related to climate change, potentially leading to a dissociation or alienation of this problem $[17,18]$.

As it has been pointed out in several different studies $[9,19,20]$, an identity process (values are general and abstract principles that one strives for in life, while self-identity reflects how one sees oneself; we will limit further discussion to the latter terminology; see [21] for a conceptual differentiation between environmental preferences, intentions and behaviour) may determine how people handle the threat of climate change. Following this line of thought-that factors such as personal values and orientations have a significant role for the choice of a response when feeling threatened-the common approach to create motivation for a green transition by focusing on 'how to formulate the message best' might be misleading. It has been pointed out that environmental messages by professional communication experts are incapable of developing the large scale mobilisation [22]. 
Thus, a main pillar of our model effort on the processing of negative information about climate change are the people's beliefs and correlated values.

By visiting several research efforts in the field of environmental psychology that investigated responses to environmental threats, we identify two significant characteristics that are reported as important drivers: (i) Climate change scepticism and (ii) and environmental self-identity. Scepticism towards climate change correlates with the belief in a just, orderly and stable world, while scepticism itself is a multidimensional construct of several types of doubts [23]. As a consequence, people with high scepticism or even complete denial of climate change show only little intention of reducing their environmental footprint when confronted with threatening news [24]. On the contrary, this information seems less convincing $[25,26]$. People who are less sceptical are positively influenced in their environmental attitude when confronted with the same information. However, attitudes like climate scepticism are not set in stone but are influenced by dynamic social processes and epistemic scepticism has a strong connection to the embedding of individuals [27].

Combining the possible influences of these two orientations of environmental identity and climate scepticism, we pursue several general research questions:

- How does the anxiety over climate change develop under constant exposure of negative climate reports? Can high amounts of negative information backfire and increase the overall climate scepticism?

- What are possible long-term effects of negative climate messaging on the pro-environmental intentions and climate scepticism? Are environmental intentions of some undermined by others that show no interest in increasing their intention to act environmentally friendly?

- How do social contagion processes on climate scepticism influence the collective scepticism of a group of interacting people? Does collective climate scepticism evolve over time and if so, in which direction?

- How does a mixed population of climate change supporters and deniers respond to climate information when interacting with each other?

- What phenomena can be observed in populations of a general low (or high) environmental self-identity? Assuming a low environmentally friendly population reacts with a lower increase of the environmentally friendly intention, can this modest rise potentially be compensated with disseminating more information about climate change in the long run? Can seldom climate information have a sufficient effect on a very environmental friendly population?

Since the empirical studies that led us to these questions are not designed as long-term studies, or deal only with either the effects of environmental identity $[9,10]$ or climate scepticism $[24,25]$, we propose a model concept that allows some hypotheses on the combined development of threat responses and climate scepticism over a longer period of time. We are of course highly interested in obtaining comprehensive in-depth studies of that topic that allow us to develop finer concepts and raise even more questions about processes sculpting the climate debate.

\section{Methods}

Social simulations serve as a fast tool to draw hypotheses about a broader context. Modelling the impact of climate messaging via computer simulations needs to address several social aspects. The micro level of individual attitudes and individual differences are crucial to implement in order to reflect personal preferences and biases. Social phenomena and effects stemming from personal interactions are important to account for group dynamics and emergent phenomena that arise on a macro level due to interactions on the micro level. Especially for the case of implementing social-psychological effects such as presented in Section 2.2, individual decision processes and social contagion processes are vital to implement in a modelling approach, a practical tool to do so is agent-based modelling. 


\subsection{Agent-Based Modelling}

The central idea of agent-based modelling (ABM) [28] is to describe a system starting from its constituent parts. These agents have certain properties and the ability to perform different actions. Since the whole system is then modelled based on those actions and interactions, the dynamic naturally emerges from the micro-scale. Agent-based modelling is possible in all scientific areas in which systems can be seen as comprised of a large number of interacting entities. Especially in the social sciences, agent-based modelling is a prominent tool and used for various applications such as tax evasion [29], holiday trips [30] and transport choices [31], and evaluation of policy decisions [32].

With respect to climate-related issues, social simulations and ABMs are important tools for understanding social behaviour. Regarding ABM, the literature shows a strong focus on social-economic and social-ecological perspectives [33-35], agricultural modelling [36,37] and adaptation processes in connection with climate policy [38-40] and migration [41,42]. Only a few social-psychological models were developed; e.g., [43] analyses the perceived resilience of an individual to changing climatic conditions and [37] implemented risk, coping, and social appraisal in agricultural adaptation processes in Sri Lanka. The process of individual adaptability on climate change via human cognition was investigated in [44]. Important work on climate scepticism in a communication network perspective to predict climate change attitudes is presented in [27].

\subsection{Model}

We like to contribute to the line of social-psychological ABM research with a model to investigate effects of climate messaging on related attitudes of individuals, e.g., climate scepticism and pro-environmental intention. In our model, individual agents have internal orientations that shape their response when confronted with information about climate change. The agent architecture is based on the aforementioned threat and defence model [12] and is backed by empirical findings $[9,10,24]$. In addition to response mechanisms to negative information, social contagion processes were implemented via interaction networks to implement agent-to-agent communication. The model dynamics describe the time development of internal states of the agents and related environmental actions.

\subsection{Agent Architecture}

For each agent, a set of internal attitudes related to climate change messaging are implemented. The agent architecture (see Table 1), i.e., the parameters describing the internal states and properties of each agent, is comprised of an anxiety state ( $a n x)$, internal orientations of both climate change scepticism ( $c c s)$ and environmental self-identity (esi), and an intention to pro-environmental behaviour (peb) that reflects the behavioural layer in correlation to the three other internal attitudes. A simple scheme of the correlations of the four agent parameter is provided in Figure 1 (top) and the mathematical representations are given in Figure 1 (left, right). When an agent receives information, an anxiety reaction $\operatorname{\Delta an} x$ is triggered, the response function is shown in Figure 1 (left). The amount of anxiety increase depends on the value of an individual's ccs (colour code) and the information impact II (x-axis), i.e., how severe or negative the information is. Without detailed knowledge of the correlation of stimuli and reactions, we assume linearity, i.e., that a stimulus of twice the impact leads to twice the anxiety. This linear response is added to the anxiety state an $x$ of the individual, which increases most for low ccs and high information impact. If no information is received, the anxiety state follows a small natural rate of decay. 
Table 1. Agent internal state and attitudes: Parameters, ranges and explanations.

\begin{tabular}{rlcl}
\hline & Name & Value & Explanation \\
\hline esi & Environm. Self-Identity & $\in[0,1]$ & Agent's individual environmental orientation \\
cCS & Climate Change Scepticism & $\in[0,1]$ & Agent's individual belief in climate change \\
anx & Anxiety & $\in[0,1]$ & Agent's internal anxiety state towards negative \\
& & & information on climate change \\
peb & Pro-Environm. Behaviour & $\in[0,1]$ & Agent's approach-motivated probability \\
\hline
\end{tabular}
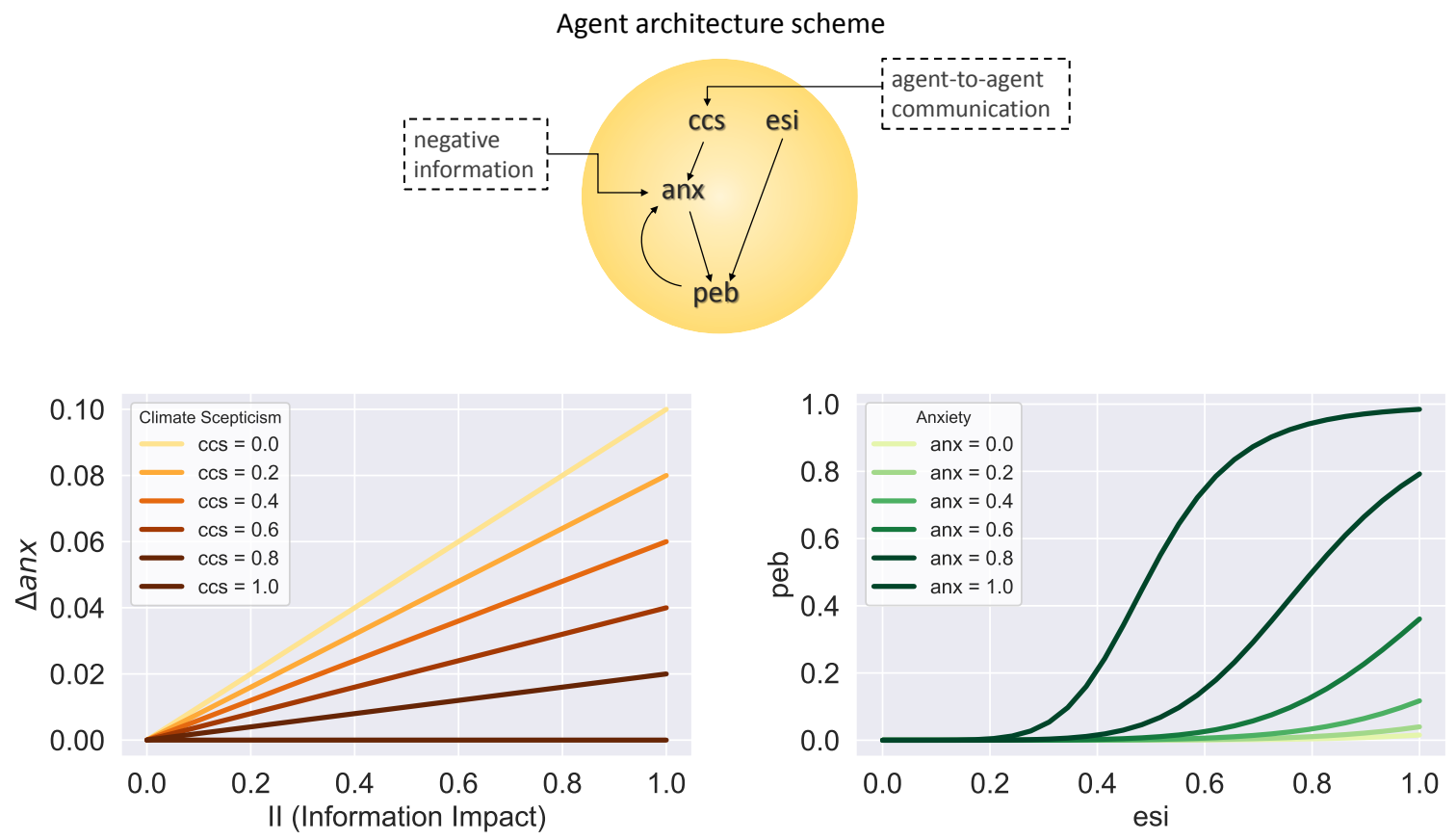

Figure 1. Agent architecture: (top) Schematic representation of direct correlations of agent parameter; (left) anxiety response $\Delta a n x$ in relation to the information impact shown for six different $c c s$ values; (right) agents peb probability in dependence of the environmental self-identity esi shown for six different anxiety states anx.

With regard to decision making and behaviour, we implement pro-environmental intentions by using a probability peb to engage in environmental friendly actions.

The pro-environmental behaviour scale [7] to classify different ecological behaviours according to the level of involvement (low to high effort) is incorporated such that high probabilities in peb correlate to higher involvement behaviours. This probability can increase or decrease according to the values of esi and an $x$ of an individual. The higher these parameters, the higher the intention and the more likely an agent responds with approach-motivated behaviour. Activating the approach-motivation leads to a decrease in the anxiety state $a n x$ and, successively, to a decrease in peb intention due to lesser anxiety. The relation of the three parameters $e s i, a n x, p e b$ is given by a Hill function

$$
H=\frac{x^{\lambda}}{h^{\lambda}+x^{\lambda}}
$$

with the hill parameter $h$ and the hill exponent $\lambda$. We use the Hill function to generate the pro-environmental behaviour probability $H=P E B$ in dependence on the environmental self-identity and anxiety $(h=(1-a n x) * 1.5+0,5, x=e s i, \lambda=6)$. The resulting probability is shown for six different anxiety states in Figure 1 (right).

To facilitate notation, we use lowercase letters when referring to parameters of single agents (esi,ccs, anx , peb) and capital letters when referring to collective means of the population 
(ESI,CCS, ANX,PEB). The mean of the initial distributions are denoted by ESIinit, CCSinit (see Table 2). They represent the initial bias of the population. By using a Gaussian distribution with a standard deviation of 0.2 we receive the following initial conditions for a simulation run: ESIinit \pm 0.2 , CCSinit \pm 0.2 . E.g., if ESIinit is small, more individuals have a low environmental identity and only a few have a high environmental identity.

Table 2. Model parameters, ranges and explanations of the full set of simulation parameters.

\begin{tabular}{|c|c|c|c|}
\hline & Name & Value & Explanation \\
\hline ESI & $\begin{array}{l}\text { Environmental } \\
\text { Self-Identity }\end{array}$ & $\in[0,1]$ & Mean population environmental orientation \\
\hline CCS & $\begin{array}{l}\text { Climate } \\
\text { Scepticism }\end{array}$ & $\in[0,1]$ & Mean population belief in or denial of climate change \\
\hline$A N X$ & Anxiety & $\in[0,1]$ & $\begin{array}{l}\text { Mean population anxiety towards negative } \\
\text { information on climate change }\end{array}$ \\
\hline$P E B$ & $\begin{array}{l}\text { Pro-Environmental } \\
\text { Behaviour }\end{array}$ & $\in[0,1]$ & $\begin{array}{l}\text { Mean population approach-motivated behaviour } \\
\text { probability }\end{array}$ \\
\hline$N$ & Population & $1000,10,000$ & Number of agents on the network \\
\hline$T_{e q}$ & Time & $2000-10,000$ & Simulation time steps until equilibrium CCS is reached \\
\hline$I R$ & Information Rate & $\in[0,1]$ & $\begin{array}{l}\text { Probability of an agent to receive external information } \\
\text { in each time step }\end{array}$ \\
\hline$I I$ & Information Impact & $\in[0,1]$ & Severity of external information received by agents \\
\hline ESIinit & Initial mean ESI & $\in[0,1]$ & $\begin{array}{l}\text { Population ESI distribution given by ESIinit } \pm 0.2 \text {, } \\
\text { remains constant }(E S I \text { init }=E S I)\end{array}$ \\
\hline CCSinit & Initial mean CCS & $\in[0,1]$ & $\begin{array}{l}\text { Initial collective CCS distribution of the population is } \\
\text { given by CCSinit } \pm 0.2\end{array}$ \\
\hline$\triangle A N X$ & Anxiety increase & $\in[0,0.1]$ & Agent's anxiety response towards external information \\
\hline- & Natural anxiety decrease & 0.01 & $\begin{array}{l}\text { Agent's continuous anxiety decay without exposure to } \\
\text { external information }\end{array}$ \\
\hline- & $P E B$ anxiety release & 0.25 & Agent's anxiety drop induced by $P E B$ action \\
\hline- & CCS learn rate & 0.05 & $\begin{array}{l}\text { Agent's maximal learn rate for social contagion related } \\
\text { to climate scepticism }\end{array}$ \\
\hline
\end{tabular}

We would like to point out that personal traits eventually have statistical correlations, so that there is a natural interdependence. In this generic approach we do not concern ourselves with the relationship between the two attributes of esi and $c c s$ and therefore treat the distributions as independent from each other. In empirical studies on climate messaging $[9,10]$ the environmental intention was treated as a sum of different tasks ranging from energy saving to waste separation. Following this approach we use a generic form of pro-environmental intention $P E B$ that does not specify a specific topic of actions and thus cannot be linked to a reduction rate of carbon emissions or similar measurements.

\subsection{External Information}

In the regarded empirical survey concerning impacts of negative climate information, fifteen statements on climate change impacts concerning Austria were presented [9]. These statements are simple and the authors refrain from using additional threatening wording (e.g., catastrophe or danger) but focus on generic statements that encompass diverse topics (e.g., future extinction of the Austrian heraldic animal, air pollution, and shift of the snow line) to outline a holistic perspective of possible regional impacts. In this setup, participants (Austrian residents) had to vote if they find a statement interesting or not and chose their top three notable statements. In alignment to the generic climate facts presented in this survey, we chose a generic version of a negative climate message to be impacting an agent population such that all agents receive the same messages but on different random points in time.

Since we are interested in the social-psychological responses on negative messages, it is sufficient to incorporate in the model the "impact' on an agent and not the information itself. The implemented 
external impact or information density can be varied in two aspects: Severity and frequency. The severity can range from strong to light and is regulated by the information impact $I I$. The messages presented in empirical work [9] would correlate to rather high values of II due to the geographical alignment of participants with the depicted regional impacts as well as the holistic nature of the combined statements that result in an impression of a complex threatening situation that affects a myriad of different domains.

The information rate $I R$ indicates the probability that an agent receives information at the beginning of a time step. This correlates to an ongoing information stream that counters a natural decay of anxiety over time due to mellowing mechanisms or avoiding the problem.

In summary, the two information parameters $I R$ and $I I$ control the information density so that we can create scenarios ranging from 'mild but frequent' to 'intensive but sparse' exposure. It is important to note that we limit the information stream to negative messages, which is only a part of the versatile climate debate that also consist of positive messages of reinforcement, motivational support and informing about successful countermeasures to limit the impact of climate change. We chose to limit this model to negative information to be able to create scenarios to observe effects of threat responses and correlated social contagion processes without any interference from additional layers of the complex debate.

\subsection{Social Interactions}

The shaping of public opinion through social interactions stems from a complex interplay between mental and social dynamics. This allows attitudes or opinions to emerge, spread and change on a population level. Agent-based modelling is particularly suitable for the study of dynamics involving heterogeneity and social contagion processes [45-49]. We use networked ABM, in which interactions are based on an underlying network consisting of $N$ agents (population).

We identify climate scepticism as the main contagious attitude with respect to social dynamics, and anxiety as a motivational impulse to participate in possible interactions about climate change. Thus, we model social contagion dynamics of climate change scepticism with a correlation to the internal anxiety level: The higher the personal anxiety the more likely it is that an agent will communicate and try to influence one of its network-neighbours, i.e., other agent's who are connected to this agent via link. If an agent is more likely to believe in climate change $(\operatorname{ccs}<0.5)$, it can reduce the scepticism of one of its link-neighbours up to a pre-defined maximum, which translates into a learning-rate. On the other hand, if an agent denies climate change to a certain degree $(c c s>0.5)$ that agent can increase the scepticism of a link-neighbour in the same way.

The model is limited to contagion of climate scepticism, while contagion processes of environmental identity and behavioural intentions are not considered. We believe that climate scepticism is the most important contagious attitude for several reasons: First, in relation to the everlasting movement of climate change denial, the contagious effect of scepticism has been shown to be highly influential among (some) members of society. Second, we believe that the denial of climate change is closely linked to the information received and less to a real and scientific understanding of the situation, which makes this dynamic even faster. Third, environmental self-identity, which involves the process of forming one's own identity, has a stronger need for experience. Therefore, we believe that the time scale of $c c s$-contagion processes is considerably faster and that social contagion processes of environmental identity are to some extent negligible for our modelling requirements.

Social contagion of environmental intentions is an additional powerful and shaping process in the public discourse on climate change mitigation. Positive messages such as "what you can do", "we can change this" or "I am doing this and so can you" are important catalysts to the social dynamics. While the primary objective of the here presented model is to simulate threat responses on negative messaging, we are very interested in adding positive information in a future research effort of a broad representation of the climate debate. However, this would imply adding different layers of reinforcement and psychological theories and extend the scope of this first investigation of that topic. 


\subsubsection{Social Network}

For readers interested in further details of the network algorithms, the underlying network topology are scale-free networks that exhibit a distribution of degrees (i.e., number of links for each node) that follows a power law. We generated this type of topology using a preferential-attachment algorithm [50]. In a second set of investigations to test the robustness of our results, spatial-proximity networks are used. These networks have a high clustering with the number of links in their spatial neighbourhood being given by the average degree $d$ to model spreading dynamics of epidemics (SIR model) [51].

\subsection{Simulations}

We use the presented model in order to investigate different aspects of climate communication under ongoing external information flow and social contagion processes over successive rounds $t$. We do the following simulations using parameters presented in Table 2. All simulations show temporal dynamics in CCS, ANX, and PEB while ESI and the information density parameters $I I, I R$ are fixed for each run.

Simulations have an equilibrium period of $T_{e q}=2000-10,000$. After reaching the equilibrium we simulate for 500 more steps in order to calculate the equilibrium as mean value. Thus, the respective simulated total time $T_{e q}$ depends on the occurrence of an equilibrium. The equilibrium state is reached when the CCS-contagion process is complete and the variations of the collective CCS are less than $10^{-3}$. For almost all simulations, we found equilibration before $t=10,000$. In the extremely rare case that a simulation did not equilibrate before $t=10,000$, we examined the results individually and found that very small fluctuations are slightly above our chosen equilibrium criterion but no trend in the CCS development was visible for the last 5000 time steps.

The model was developed in NetLogo 6.0.4 [52] and the evaluation of data and generation of figures was done in Python 3.6 (open source, https:/ /www.python.org). The validation of the model results include face validity through graphical display (animation) and visiting of collective and selected agent time lines (graphical representation and tracing), extensive stochastic comparison to track inconsistencies (internal validity), and a sensitivity analysis via parameter variation that showed a high robustness of the behaviour over reasonable parameter ranges. The validation procedure encompasses all techniques which are relevant for our model setup (see [53]).

\section{Results}

Using the proposed model to evaluate scenarios, we can now investigate both favourable scenarios (i.e., scenarios with high environmentally friendly behaviour) as well as unfavourable scenarios. That way, we can identify the conditions that are necessary for pro-environmental behaviour. For this, we present different analyses of our model, which can be divided into studies on

1. the development of the distribution of different agent parameters over time,

2. the equilibrium results of these parameters as collective mean states,

3. the heterogeneity of behaviour in the population and divergence into strongly opinionated subgroups, and

4. the role of information density to activate a permanent environmental intention increase.

For the time dynamics within a population, we explain the evolution stages of agent's opinions and actions. With the two internal orientations esi and ccs being responsible for the bias of populations on a macro scale (ESI,CCS), we perform a rigorous parameter analysis to contrast results for different initial biases. This allows us to compare the behaviour of populations of weak to strong environmental self-identity and low to high climate change scepticism. To test the influence of the interaction network, we compare these results on two different network types (scale-free networks and spatial-proximity networks). To give more insight into trends within a population such as the formation of strong 
opinionated subgroups, we compare the behaviour of climate change 'believers' and climate change 'deniers' as a sub-investigation of the parameter analysis of the internal orientations. In order to illustrate the influence of the information, we compare scenarios with low, medium and high information density. After discussion of the main effects of the information density on the dynamics, some minor effects regarding the temporal development and similarities under 'mild but frequent' to 'intensive but sparse' exposure are included to complete the picture.

To simplify the understanding of the results we distinguish the parameter space of initial ESI,CCS in four quadrants A, B, C and D. Each quadrant represents populations with a specific bias. Quadrant A: More sceptical and less environment-oriented, quadrant B: More sceptical and more environment-oriented, quadrant C: Less sceptical and less environment-oriented, and quadrant $D$ : Less sceptical and more environment-oriented. The balanced or neutral population of $E S I=0.5$ and $C C S=0.5$ is centred in-between the quadrants.

\subsection{Time Evolution}

The general dynamical response processes under continuous exposure of negative climate messages evolves on a population level as follows: Each simulations starts with no anxiety $A N X=0$ and, therefore, no probability to perform PEB. The continuing exposure to information of rate $I R$ and impact $I I$ increases the anxiety level which is then followed by a collective $P E B$ response. This response is delayed due to the necessity of a certain anxiety level to emerge within the population. Additionally, the initial normal distribution of the scepticism with mean CCS transforms over time into two subgroups of supporters and deniers of climate change messages. This effect is also delayed for the same reason as the $P E B$ response.

Figure 2 shows this dynamical processes for a balanced population under exposure of medium information density: First, an increase in anxiety is visible while the PEB probability and collective CCS distribution are steady. With a delay, the scepticism divides and the normal distribution with mean CCS is dispersed. Strong believers $(\operatorname{ccs}<0.1)$ and strong deniers $(\operatorname{ccs}>0.9)$ become more frequent while the rest of the opinion range is uniformly present. While this process continues, the intention for pro-environmental behaviour is not affected. At $t=2000$, an equilibrium state is reached and shows a pronounced heterogeneity especially for the anxiety levels and scepticism of climate change. The population is separated into two strongly opinionated groups of unequal group sizes. The overall high anxiety is due to the larger group of strong believers. However, the environmental intention is significantly lower than the correlated anxiety level and this example results only in a small impact of negative climate information by medium density.

To get more insight into the role of the information density we show the evolution of the collective anxiety level and the correlating pro-environmental intention increase for low, medium and high information density in Figure 3. The results represent the mean (line) and standard deviations shaded areas (only visible for PEB at high density) of 20 simulations for each density and populations of $N=10,000$ agents. For neutral populations, a notable notable effect of the information is visible: While the anxiety levels for medium and high information are similar, the environmental intention differs greatly. Here, the correlated approach-motivated behaviour at high information density is more than doubled compared to the medium density case. Another striking aspect is that, at low densities, anxiety rises less sharply and no impact of the information on the population's environmental intentions is visible. It is important to note that the stable states are due to an ongoing information flow which, if varied, would produce a more dynamical response over time. If the information stream is lessened or discontinued, the intention increase would successively decrease. Similar results can be obtained by using smaller populations ( $N=1000$, see Figure A1). No major scaling effects by variations in population size have been observed. 


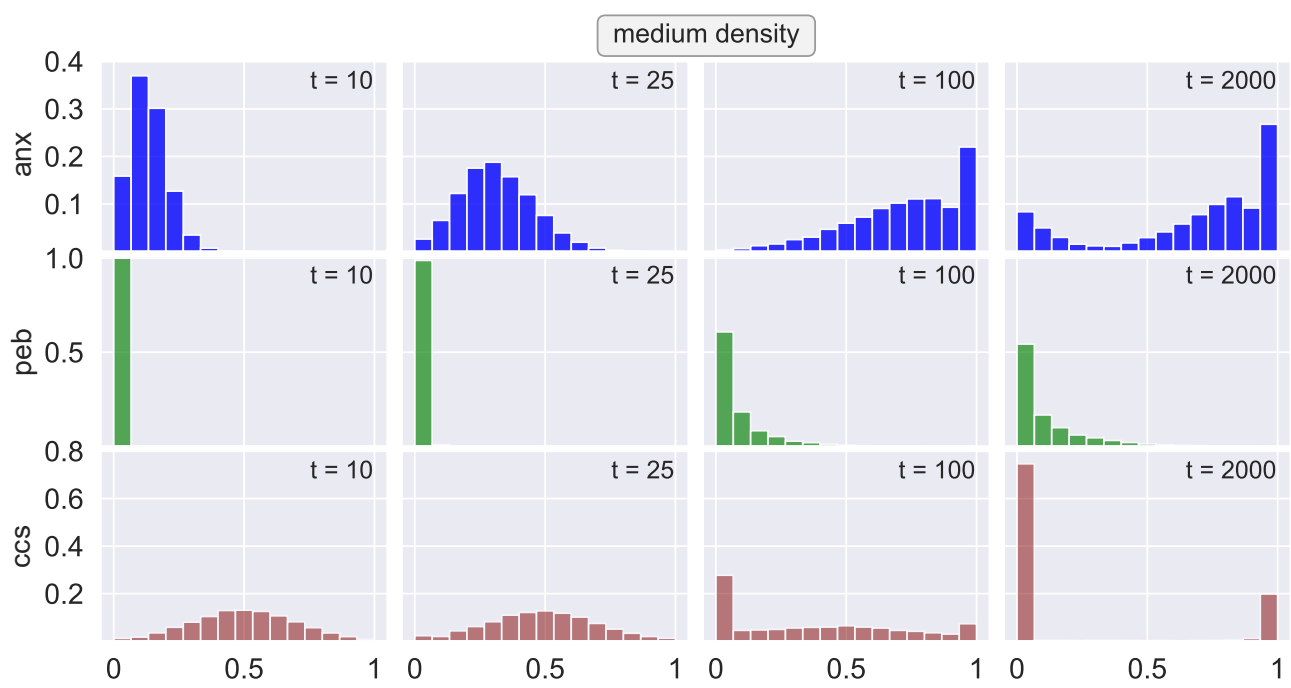

Figure 2. Temporal evolution of heterogeneous behaviour: Evolution stages of peb (green), anx (blue) and $\operatorname{ccs}$ (brown) shown in four chronological time steps $t=10,25,100,2000$ (from left to right). The balanced population of initial distributions around ESI $=0.5$, CCSinit $=0.5$ is exposed to a medium information density, representing the moderate case of development patterns. Parameter $I I=0.5, I R=0.5, N=10,000$.
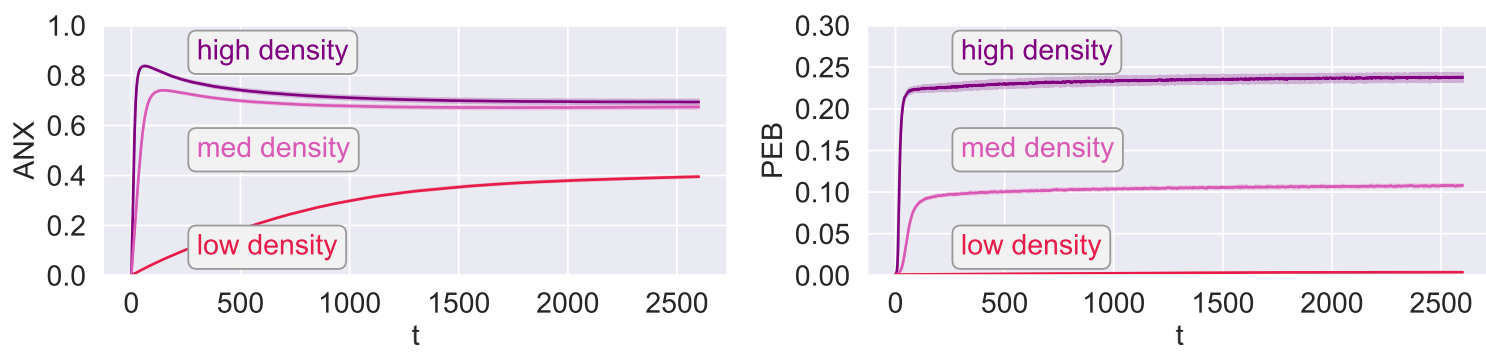

Figure 3. Time evolution of collective anxiety $A N X$ (left) and collective pro-environmental behaviour $P E B$ (right) for three different information densities: Low $(I I=0.1, I R=0.1)$, medium $(I I=0.5, I R=0.5)$, and high $(I I=0.9, I R=0.9)$. Results show mean values (lines) and standard deviations (shaded area) of 20 simulation runs. Parameter: $N=10000, E S I=0.5$, CCSinit $=0.5$.

To summarise the results on the time dynamics, we find that:

- Negative climate information produce a variety of anxiety reactions inside a population of different individuals from no increase to a strong increase.

- An increase in the collective anxiety does not strictly lead to an increase in the environmental intention.

- Activation of approach-motivation that increases environment-friendly intentions is delayed and emerges after sufficiently high levels of anxiety have developed.

- The social dynamics produce a separation of two strongly opinionated groups of believers and deniers of climate change.

- Continuous information streams play an important role to successfully increase the environmental intention. If not enough or discontinuous information is provided, no significant or stable increases are observed. 


\subsection{Dependence on Environmental Self-Identity and Climate Change Scepticism}

The initial configuration of the population, i.e., whether it is a population with low or high environmental values or climate scepticism (four quadrants), is decisive for the outcome of the behaviour patterns. To test the dependence of the environmental intention (PEB probability) on the internal parameters (esi and $c c s$ ) of the agents, we perform a complete parameter variation, as shown in Figure 4. Here the initial distributions of both parameters are varied with ESIinit $=E S I \in[0.01,1]$ and CCSinit $\in[0.01,1]$, reflecting the four population quadrants $\mathrm{A}, \mathrm{B}, \mathrm{C}$ and $\mathrm{D}$. The results are given for the mean collective anxiety ANX (left column, Figure 4) and the mean collective PEB probability (right column, Figure 4). The initial mean parameters ESI,CCSinit are reflected by the $x, y$-axis. Results are displayed for the mean of 10 (high density) and 5 (low density) simulation runs for each set of parameters.
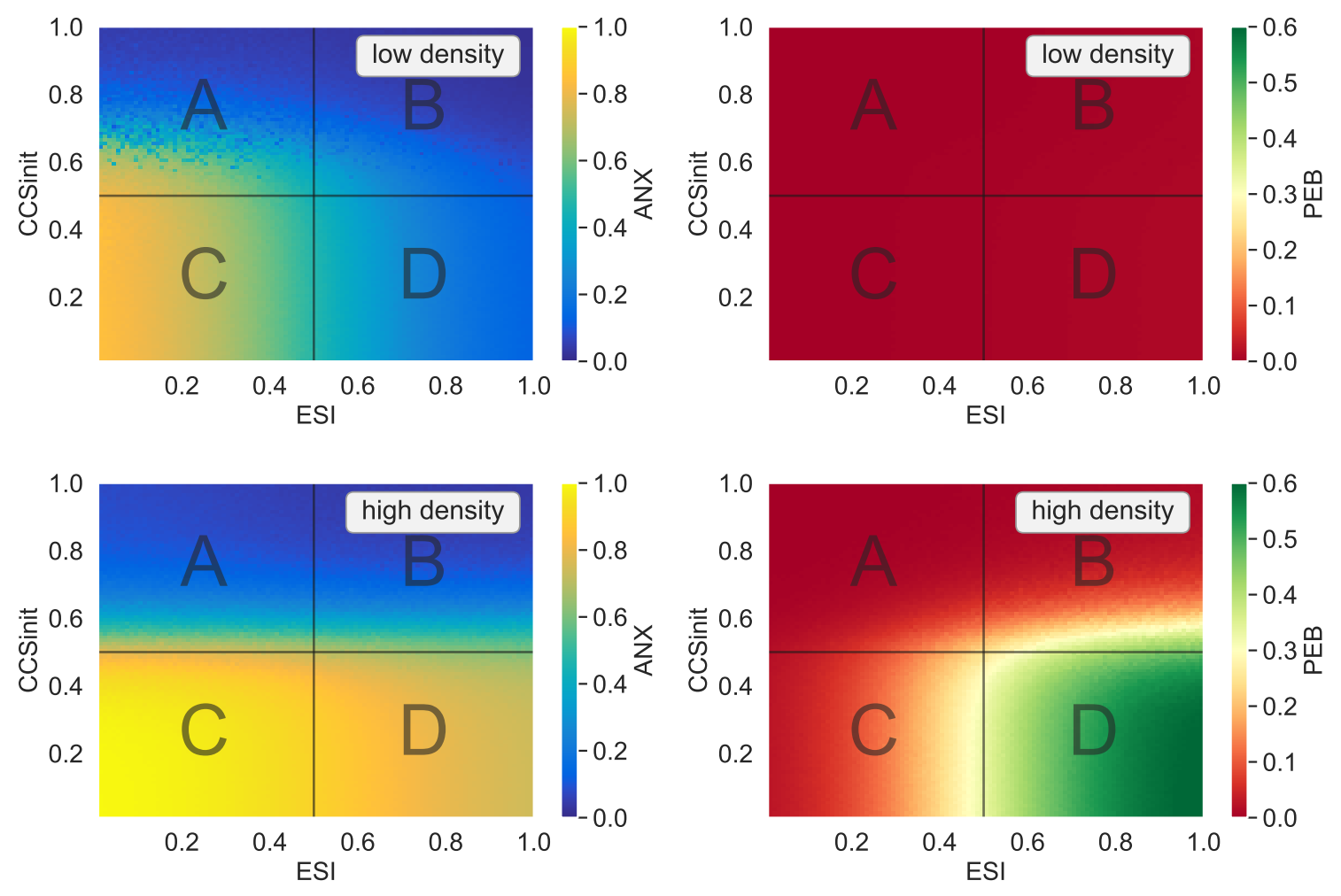

Figure 4. Dependence on population bias: Exploration of the initial orientations ESI and CCSinit on the population behaviour for (top) low $(I I=0.1, I R=0.1)$, and (bottom) high $(I I=0.9, I R=0.9)$ information density. Results shown for (left) collective anxiety $A N X$, and (right) pro-environmental behaviour $P E B$, average taken over 5 (low) and 10 (high) simulation runs. Colour code as shown in the colour bar. Parameter $N=1000$, scale-free topology.

We observe the following phenomena: In the case of low information density (top), the collective anxiety is increased only in quadrant $C$, while the other quadrants exhibit rather low anxiety levels. For all quadrants the correlated PEB probability increase is not significant $(\approx 0.006)$ even for the optimal internal values of quadrant D (populations with ESI $>0.9$ exhibit an increase of 0.012 , not visible in the colour code). Thus, we observe no significant influence of climate messaging under low information density on the approach-motivation for any population.

In the case of high information density (bottom) several effects occur: First, a correlation of the collective anxiety to the collective scepticism is visible. Populations leaning towards climate scepticism located in quadrant A, B $(C C S>0.5)$ exhibit low anxiety. Populations leaning towards supporting a believe in climate change located in quadrant $\mathrm{C}, \mathrm{D}(\mathrm{CCS}<0.5)$ show higher anxiety 
levels, this increase is even stronger in quadrant $C$. Second, we observe a positive correlation of the anxiety level and favourable PEB increase, however, only for populations with high ESI $>0.5$ and low scepticism CCS $<0.5$ which are located in quadrant D. Populations of other quadrants show no significant increase in the PEB probability and thus are considered unfavourable. We conclude that the internal orientation bias of the population plays a major role and cannot be outperformed by intensive information flow.

Some additional information about the robustness of these results in regard to the network modelling are provided in the following. The results from Figure 4 are generated using scale-free networks, which represent a common network topology for societal processes. To test the robustness of our results towards other network types, we explore the same model dynamics on spatial-proximity networks, the results are shown the Figure A2. By switching to a different topology, the model output remains similar: Quadrant $A$ and $C$ do not show PEB responses and quadrant $D$ reacts approach-motivated with increased $P E B$. In quadrant $B$ an improvement of $P E B$ responses takes place, dividing the quadrant into two areas with and without PEB response. To summarise the results on population biases, we conclude:

- Results for low information dissemination do not indicate promising developments. None of the populations respond with approach-motivation to sparse messaging, even under the best conditions of strong believe in climate change and sufficient environmental self-identity among the individuals.

- For high supply of information, only populations under the best conditions of low scepticism and high environmental identity react with enhanced approach-motivation. However, these populations are not able to fully resolve their anxiety and remain anxious.

\subsection{Climate Scepticism Dynamics}

Another important aspect are changes in climate scepticism with regard to the amount of information a population is exposed to. To illustrate the social contagion processes on CCS, we examine the relative changes between initial climate scepticism (CCSinit) in a population and the resulting equilibrium state CCS, shown in Figure 5 (left). Values of $C C S$ below the dashed line indicate a decrease in respect to the initial configuration, while values above CCS indicate an increase in collective CCS.
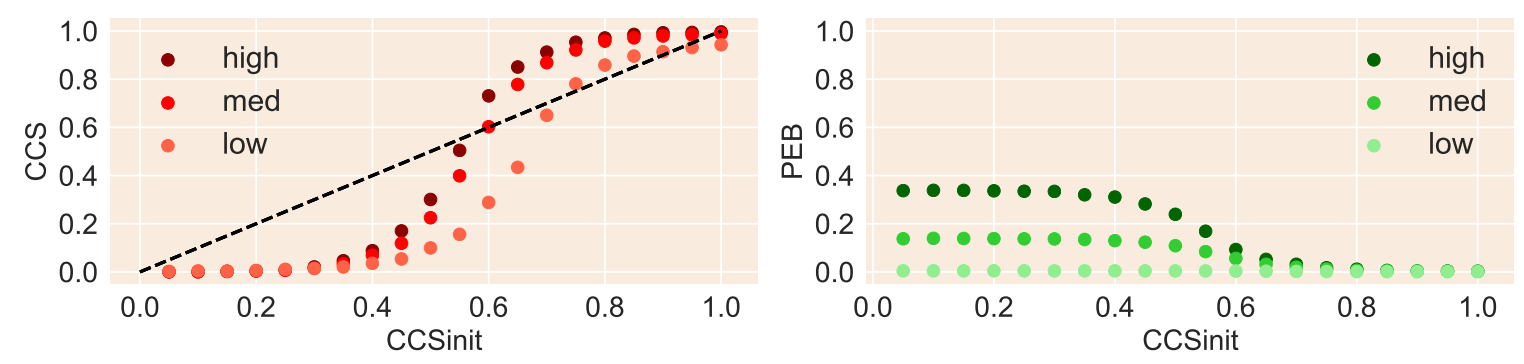

Figure 5. Dependence on initial climate change scepticism: Correlation of initial CCSinit and resulting (left) climate change scepticism CCS and (right) pro-environmental behaviour PEB. Results shown for three different information densities (low, medium, high) as shown in the legend. Parameter: $N=1000, E S I=0.5$.

We show the results for three scenarios of the information density: Low $(I I=0.1, I R=0.1)$, medium $(I I=0.5, I R=0.5)$, and high $(I I=0.9, I R=0.9)$. Interestingly, the higher the information density, the stronger the CCS increase for same initial configurations: Using the balanced population at CCSinit $=0.5$ as an example, we observe a reduction of the of CCS for all information densities, however, this reduction is less pronounced the higher the information density. Moreover, inspection of the correlated timelines (not shown) revealed that the time dynamics can undergo 
intermediate states. For the high density case, the final decrease in CCS was preceded by a small intermediate increase of $C C S \approx 0.6$ which ultimately declined towards $C C S \approx 0.3$.

Next to the evolution of scepticism over time, we are particularly interested how this affects the increases in pro-environmental intentions of the population. To highlight differences in the $P E B$-increase for the same scenarios of low, medium and high information density, we show the collective mean $P E B$ probability in Figure 5 (right). It is evident that populations with high initial scepticism $(C C S>0.7)$ are hardly influenced by any amount of information. On the contrary, populations with a strong bias towards believing into climate change $(C C S<0.3)$ show a strong correlation with the information and respond proportionally to the information density. However, there are some notable changes in the group in between $(0.3 \leq C C S \leq 0.7)$. On the one hand, the resulting CCS display a correlation with the available information and rise the higher the information density (red colour code, Figure 5 left). On the other hand, there is also a positive correlation of the information stream for the PEB response, which also increases the more information is provided (green colour code, Figure 5 right).

Summing up the results on the varying degree of collective scepticism and subsequent development in context of negative climate reports, we conclude that:

- Highly sceptic societies tend to increase their scepticism when being confronted with negative information about climate change. Neutral and supporting societies decrease their scepticism further.

- Neutral societies can exhibit an intermediate increase in climate scepticism but ultimately, the mean climate scepticism shows a stable reduction.

- The effect of scepticism reduction is diminished the more negative information is disseminated. At the same time, the environmental intentions are increased the more information is present. Thus, in terms of an overall effect of negative climate messages, a higher density is preferable, but positive effects are shortened by a stronger expressions of climate denial.

\subsection{Believers and Deniers}

The time evolution of the collective CCS leads to a transformation of the initial normal distribution around the initial mean value CCSinit into distinct groups of low and high climate scepticism (as presented in Figure 2). Since the agent architecture does not include intelligent actions, i.e., agents cannot change their opinions by logical thinking when presented with convincing evidence, this sharp division is a result of the social contagion and a typical phenomenon for generic agent-based modelling without noise. Adding more layers to the social contagion processes might produce softened results, yet the results, using only CCS-contagion hold a good indicator of some fundamental dynamics inside a population.

In order to further investigate this division and its consequences, we categorise the population into two groups. The 'believers' are those whose $c c s$ is below $50 \%$, the 'deniers' are the agents whose ccs is above $50 \%$. We study the parameter dependencies and different behaviour patterns of both groups over the parameter space of internal orientations ESI, CCSinit (see for comparison Figure 4).

Figure 6 shows different relations and dependencies in respect to both collective orientations ESI and CCSinit for a high information density. We show an evaluation of group parameters for both groups of believers (green) and deniers (brown). To capture the dependence on both internal orientations-environmental self-identity and climate scepticism-for both groups in the same graph, we depict the variable (ESI or CCSinit) with the stronger effect on the respective parameter $\left(N_{B, D}, C C S, A N X, P E B\right)$ on the x-axis and the less pronounced dependence on the other variable is depicted by the mean (line) and shaded area (standard deviation). In this representation, curves with a small standard deviation show that the secondary dependence is negligible. Here the group sizes $N_{B}, N_{D}$ and the resulting group scepticism CCS exhibit a stronger dependence on the initial scepticism CCSinit (Figure 6 (top)). This is inverted for the group anxiety ANX and group 
environmental intentions $P E B$ which show a stronger dependence on the initial environmental self-identity (Figure 6 (bottom)).
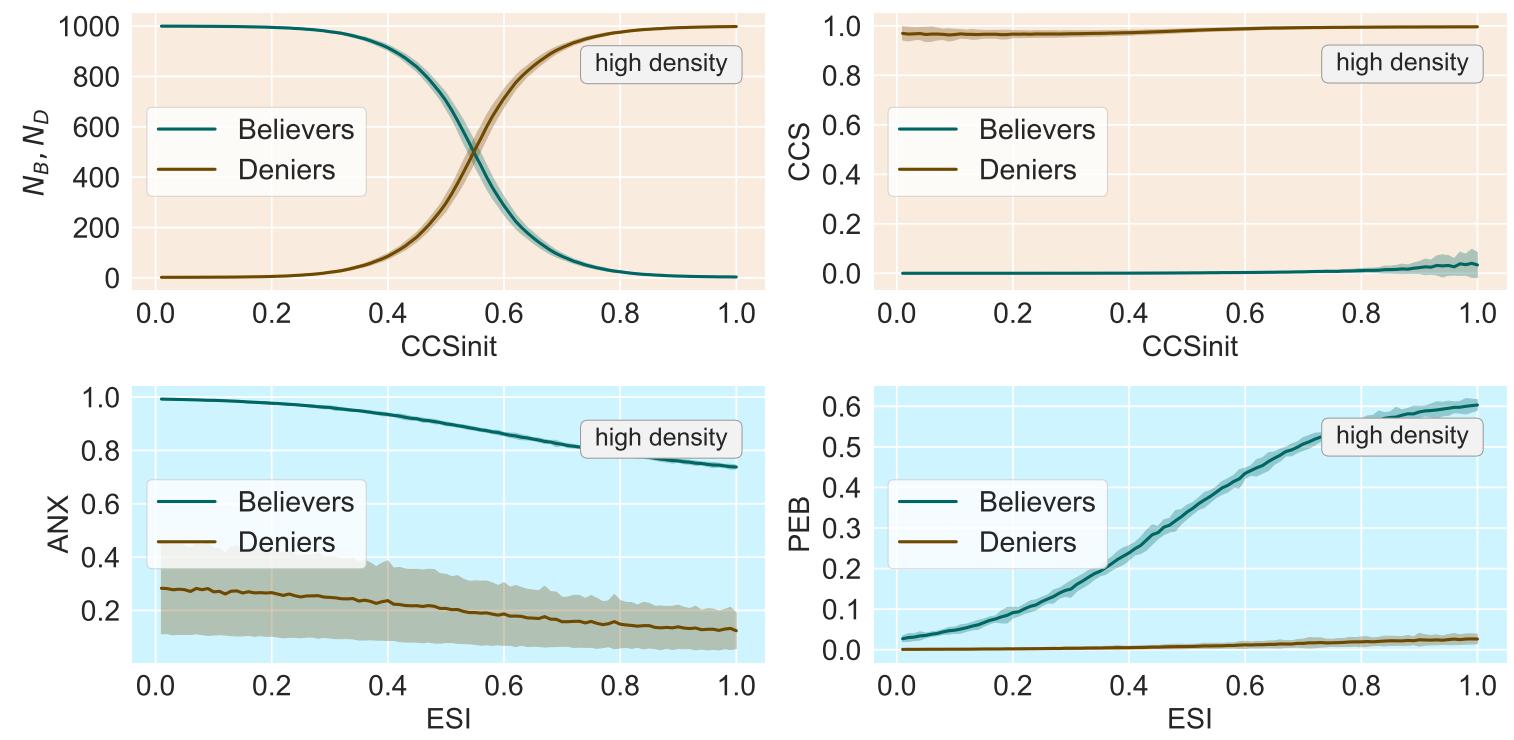

Figure 6. Believers and deniers: Dependency of both groups on the internal orientations ESI, CCS in the high information density scenario. (top left) Population share sizes $N_{B}, N_{D}$, (top right) CCS equilibrium values, (bottom left) anxiety $A N X$, and (bottom right) pro-environmental behaviour $P E B$. Parameter: $N=1000, I I=0.9, I R=0.9$

One of the most important properties are the group sizes, $N_{B}$ of the believers and $N_{D}$ of the deniers. There is a naturally correlating relationship of the resulting group sizes that is proportional to the initial mean of the normal distribution of scepticism CCSinit. The higher CCSinit the higher the number of $N_{D}$ and vice versa (see Figure 6 (top left)). For higher the initial scepticism, we observe a smooth decay of believers in favour of deniers. The dependency of group sizes on the environmental identity is negligible due to the narrow shaded area of each curve.

Another important finding is that the resulting scepticism CCS is uncorrelated to both internal orientations, as shown in Figure 6 (top right). Both groups display a strong opinion for all values of CCSinit (constant over the x-axis) and ESI (small shaded area).

Figure 6 (bottom) shows the investigation for believers and deniers of the group anxiety and group pro-environmental intent in respect to both internal orientations CCSinit, ESI. Here the perspective on CCSinit and ESI is inverted: The x-axis depicts the correlation to ESI while identical CCSinit are averaged resulting in the shaded area around the mean line. This inversion is feasible due to the natural dependence of $P E B$ on the environmental self-identity. Indeed, only the anxiety of the deniers displays both dependencies of ESI and CCSinit. We observe an overall higher anxiety level for the group of believers than for deniers. Additionally, the anxiety of deniers shows a secondary strong dependence on the initial scepticism (large shaded area).

It should also be noted that believers are exclusively contributing to $P E B$ while deniers do not respond with any increase in PEB. Therefore, favourable results of quadrant D (see Figure 4 (bottom right)) are solely due to the believers, which are motivated to respond with PEB. The deniers can be regarded as free-riders [54], who benefit from the actions of others as they are not willing to contribute. Thus, the group sizes $N_{B}, N_{D}$ are a major influence on the success of the whole population.

In the appendix, this investigation is shown for low information density (Figure A3). Additionally, we provide the results on the complete parameter-plane of ESI and CCSinit for both groups of believers and deniers on which this evaluation is based on (low density: Figure A4, and high density: Figure A5). 
To summarise our results on opinionated groups, we find that:

- The numbers of believers and deniers correlate strongly to the initial bias on climate scepticism before climate messages are distributed.

- Both groups show highly pronounced opposing attitudes on climate change.

- Believers demonstrate a stronger influence of climate statements by strongly increased anxiety levels. This increase is not as pronounced as pronounced the higher the group's environmental self-identity which reflects positive coping strategies of the group of believers.

- Deniers also react to the climate statements by increases in anxiety, but are far less affected.

- Only the group of believers can be encouraged to improve their pro-environmental intention through negative climate messaging. Deniers can be regarded as free-riders who exploit the efforts of believers to change their behaviour towards climate change.

\subsection{Effects of the Information Density}

In this section, we would like to highlight and summarise the main findings on effects of the information density that have been presented in Figures 2-6. The implemented information stream represents negative climate messages that provoke threat responses of agents. All agents receive the same information and it is due to their internal orientations how they cope with the threat. The primary effect observed is an increase of the overall anxiety which can lead to a positive shift in pro-environmental intentions. The actual increase in environmentally friendly behaviour is subject to several factors. In general, the confrontation with the consequences of climate change does not increase the pro-environmental intent unless several conditions are met:

1. The amount of information has to be large enough to encourage approach-motivated actions. Otherwise the anxiety increases without sufficiently promoting pro-environmental behaviour changes.

2. The majority of the population should consist of individuals with well-developed environmental self-identities. Otherwise the lack in motivation hinders individuals to respond appropriately with environmental friendly behaviour change.

3. The majority of the population should strongly believe in climate change. Otherwise free-riding of climate change deniers reduce the overall success of the population.

An important side effect correlating to the information density is an increase in the public scepticism. The more information is provided the larger the resulting group of climate deniers. Encouragingly, environmentally friendly intentions are also enhanced when more information is provided. This effect more than compensates the negative effects of increasing scepticism, leading to a favourable outcome if the other two conditions, 2 and 3, are met.

We would like to add two minor aspects of the impact of information density to the discussion to complete this picture. First, the time dynamics of contagion processes are slow for low information density. This is caused by a lower probability of participating in communication between agents when the overall anxiety levels are low. The higher the anxiety, the more discussion about climate change take place which corresponds to faster dynamics in the population.

Second, we investigate the influence of the information rate and information severity. For this it is of interest if the time dynamics (duration towards equilibrium and equilibrium state) vary for different scenarios from 'mild but frequent' to 'intensive but sparse'. Here we find only a slight difference in the resulting states and evolution times until equilibrium in favour of the information rate $I R$. Since the difference is $\sim 1 \%$, we do not draw any further conclusions.

\section{Limitations and Future Research}

In order to implement the social-psychological theory of threat and defence [12] in a networked $\mathrm{ABM}$, we chose several simplifications to obtain a generic model that can be further extended in future 
research efforts. Possible extensions or improvements are given in regard to (1) agent architecture and social contagion processes, (2) data on internal orientations (environmental self-identity, climate scepticism) and social contagion surveys, (3) information flow and information processing, and (4) including anxiety release via symbolic defensive behaviour, to name but a few. In order to deal with the questions arising from this work in further detail, we will work on the aspects mentioned below as described below:

(1) Further development of the agent architecture is provided, e.g., by the Belief-Obligation-Desire-Intention BOID architecture [55] where social agents are capable to resolve different conflict types within or among informational and motivational attitudes.

(2) We are currently designing a survey to verify the here presented model results, including aspects of environmental orientation, climate scepticism, a measurement of threat levels and anxiety, as well as social processes (e.g., the willingness to engage in the climate debate or be influenced by others regarding the climate scepticism or pro-environmental intention). We are interested in verifying the here presented results and in a second step to implement the survey as input-data.

(3) In addition to the here presented results on negative messages, widening the discussion to positive message streams is feasible. This includes additional support mechanisms for the contagion of environmental intentions. Furthermore, implementation of a direct influence of scepticism through convincing or persuasive information represents a meaningful extension of the model.

(4) The model represents a closed system, where an agent's only option to reduce anxiety is by approach-motivated behaviour or small natural decrease when not confronted with negative information. We are looking forward to conceptualise a model version that takes into account the anxiety reduction of the resulting symbolic defensive behaviour that manifests itself, for example, as ethnocentrism or other discriminatory behaviour [9].

With this in mind, we intent to further improve the model's capacities in the near future and to provide a supporting tool for critical reflection on the key challenges in connection with climate change mitigation. We would like to emphasise that our understanding of social ABM at the current state does not include predictive power, but the nature of the model as presented in this work serves for scenario design and the purpose of enrichment of the discourse.

\section{Conclusions}

This study investigates long-term effects and scenarios for the climate debate via computational simulation by drawing from the social-psychological perspective of threat and defence research. We developed an agent-based model were individuals are subsequently exposed to negative climate messages. The exposure of threatening information causes an anxiety response of individuals which then can be released by approach-motivated pro-environmental behaviour. An important aspect of how individuals deal with the presented information are their internal orientations, namely the environmental self-identity and climate change scepticism. Populations with high environmental identity and low climate scepticism perform best, but we found that they are only sufficiently motivated to increase their environmental intentions under ongoing high intensity of climate messages. A side effect that occurred when introducing more negative messages was a subsequent increase in climate sceptics who reduced the overall success of increasing the environmental intentions of the believers. We would like to highlight some of our most important insights of the model and discuss them on a broader perspective:

An increased anxiety about climate change is not necessarily associated with greener lifestyles.

Societies that are leaning towards believing in the consequences of climate change respond to threatening statements about climate impacts with increases in anxiety. However, it depends strongly on the overall environmental self-identity if they respond with approach-motivated behaviour. Anxious societies that are at the same time not encouraged enough to respond with environmental 
intentions to reduce the build-up anxiety will stay anxious over time. In real societies, this holds the risk that people resort to an alternative way of dealing with anxiety, namely by showing symbolic defence responses (backfiring effects) such as ethnocentrism and looking more negatively at multiple groups, including criminals, overweight, or unattractive people [9]. This social meta-mechanisms are not included in the model.

Providing sparse information about climate change impacts inevitably lead to unfavourable scenarios.

None of the modelled societies showed a willingness to change behaviour through a higher environmental intention, if the climate messages were not particularly severe and at the same time propagated frequently. The problem becomes even more challenging as we have observed that the number of deniers and sceptics is rising as climate reports increase. We did not find an optimal solution but good solutions came with secondary backfiring effects. Now pursuing this line of thought that the number of deniers can increase when broaching the discussion on climate change, we expect that adding misinformation-which represents well-known part of the current public debate-can lead to contagion processes towards even stronger climate denial.

High information density about climate change is not necessarily correlated with favourable scenarios.

Not all scenarios of population biases showed good results in the pro-environmental intentions, even under high information density. In terms of climate scepticism, neutral societies without a bias in scepticism evolved towards less scepticism, while this trend was dampened the more information was distributed. While climate denial is a likely factor when societies barely move forward in their green transition, the people's environmental preferences, intentions, and behaviour are strongly related to their environmental self-identity. Since approach-motivation can be empowered by personal goals derived from internal orientations, a social process towards higher environmental identities might be a key component to increase people's motivation towards choosing greener lifestyles.

The number of climate change believers is important for the success of a population, while at the same time climate change deniers are free-riding.

We observed a partition in two subgroups of opposing attitudes on climate change. While the group that supports climate messages is open to increase their environmental intention, the climate deniers will ultimately show no response when confronted with climate change impact. Our simulations showed that groups of climate deniers will form even under the best circumstances of the population bias and were not persuaded by the opinion of the majority. Even if a society manages to sufficiently increase their environmental intentions, climate deniers would benefit from the actions of others without contributing.

Initial high values on climate scepticism hinder the population to develop anxiety while the minority of believers are highly anxious but muted by the denying majority.

If the majority denies climate change, a small group of anxious supporters of climate messages remained. This is similar to the opposite case when the majority of the population supports climate messages, where a small group of robust climate deniers remain. While the anxiety levels of the modelled deniers do not increase due to climate messages, whether they are a minority or the majority, we believe that in real societies, anxiety of a minority group might build up but due to different societal mechanisms e.g., from approval and membership, and might contribute to opinion dynamics as well.

\section{General Discussion}

Drawing a general conclusion on the observed collective behaviour of the modelled population, we find that the conditions under which a transition to greener lifestyles takes place are very narrow when considering threatening information only. In order to motivate the population, an explicitly high ecological identity alone is not sufficient. In addition, an ongoing high information load, which, 
realistically, cannot be achieved in real-world systems, would be required. We believe that this indicates that a self-regulated transition, meaning that individuals choosing voluntarily to change behaviour permanently, is rather unlikely. This leads us to conclude that systemic regulation, including environmental laws, sanctions for exceeding footprints, and financial incentives is needed to enable a transition towards sustainable societies. This portrayal is model-based only and might be rather pessimistic, since we did not include motivators, such as hope in the context of climate change [56] or overcoming scepticism, for example, through education [57] and decreasing the costs and efforts to 'go green' [58].

Furthermore, we are only observing reactions towards negative information while the climate debate is also fuelled by positive messages such as 'what you can do' or 'I am doing this and so can you' which are important catalysts to the social dynamics and may pose strong counter to the here presented threat-based responses. Negative climate messaging is only one side of the coin that fuels the climate debate. We believe that education, positive reinforcement and of course identity building by telling stories about 'green pioneers' and societal movements such as 'Fridays for Future' [59] lead to different societal dynamics as we observed in our model about threat reactions.

It has been shown that there is a connection between attitude toward nature and attitude toward environmental protection [60]. We believe that increasing environmental identities is a key approach to catalyse positive societal dynamics that is often overlooked when concentrating on the vast pile of information and endless discussions around climate aspects. Aside the climate debate, there are many options to strengthen environmental identities not only through education [61] but by experiencing nature [62] to promote higher appreciation and deeper personal connections. As shown previously, ecologists are required to work together with urban planners, developers, architects and other stakeholders including members of the local community to improve upon the interactions between people and nature [63].

In any case, in order to reach the goal of sustainable lifestyles through climate messaging, telling the consequences of climate change is only part of the solution. Maybe more importantly, we need to think about how to diversify information and introduce the psychological perspective on sustainability and sustainable development to facilitate this transition [64]. This kind of research is highly complex and requires an interdisciplinary approach, but it is absolutely paramount to tackling the challenge of a green transition.

Author Contributions: Conceptualisation, M.L.K.; methodology, M.L.K. and G.J.; software, M.L.K.; validation, M.L.K.; formal analysis, M.L.K.; investigation, M.L.K.; data curation, M.L.K.; writing - original draft preparation, M.L.K.; writing-review and editing, M.L.K. and G.J.; visualisation, M.L.K. All authors have read and agreed to the published version of the manuscript.

Funding: The authors would like to thank the University of Graz for covering the publication fees. M.L.K receives a student grant by Steiermärkische Sparkassen and the University of Graz, Austria.

Acknowledgments: The authors would like to thank Manfred Füllsack for his support. Open Access Funding by the University of Graz.

Conflicts of Interest: The authors declare no conflict of interest. The funders had no role in the design of the study; in the collection, analyses, or interpretation of data; in the writing of the manuscript, or in the decision to publish the results.

\section{Abbreviations}

The following abbreviations are used in this manuscript:

$\begin{array}{ll}\text { ABM } & \text { Agent Based Model } \\ \text { BAS } & \text { Behavioural Approach System } \\ \text { BIS } & \text { Behavioural Inhibition System } \\ \text { GHG } & \text { greenhouse gases }\end{array}$




\section{Appendix A. Additional Figures}
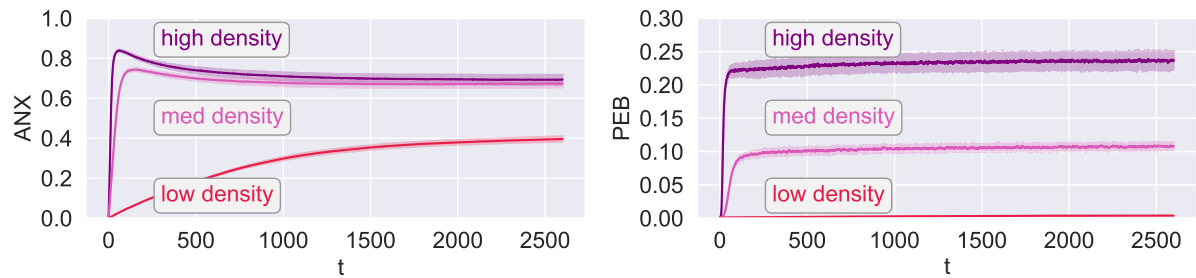

Figure A1. Time evolution of population of size $N=1000$ : (left) Collective anxiety $A N X$, (right) and pro-environmental behaviour $P E B$ for three different information densities with low $(I I=0.1, I R=0.1)$, medium $(I I=0.5, I R=0.5)$, and high $(I I=0.9, I R=0.9)$ given by the colour code. Results show mean values (lines) and standard deviations (shaded area) of 20 simulation runs. Parameter: $N=1000, E S I=0.5$, CCSinit $=0.5$.
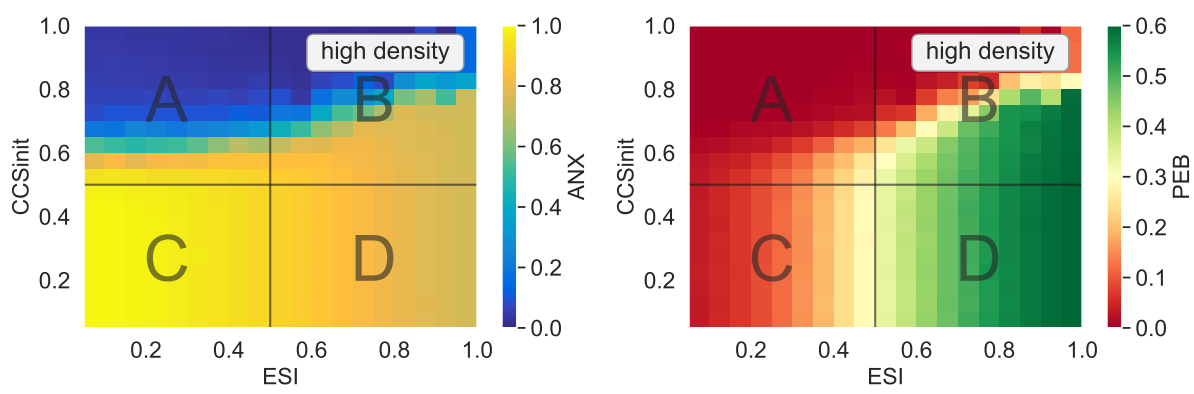

Figure A2. Dependence on population bias with spatial-proximity networks: Exploration of the initial orientations ESI and CCSinit on the population behaviour for high information density. Results for (left) collective anxiety $A N X$, and (right) pro-environmental behaviour $P E B$, average taken over 20 simulation runs. Colour code given in the colour bar. Parameter $N=1000, I I=0.1, I R=0.1$, $T_{e q}=2000-10,000$, average degree $d=6$.
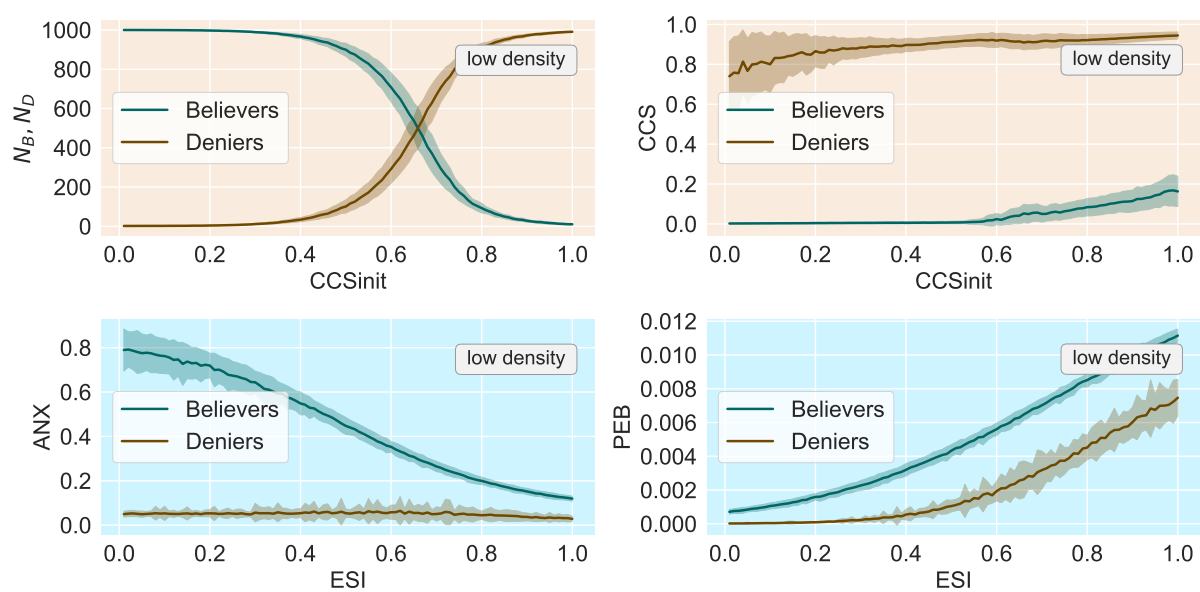

Figure A3. Believers and deniers: Dependency of both groups on the internal orientations ESI, CCS in the low information density scenario. (top left) population share sizes $N_{B}, N_{D}$ (top right) CCS equilibrium values, (bottom left) anxiety $A N X$, and (bottom right) pro-environmental behaviour $P E B$. Parameter: $N=1000, I I=0.9, I R=0.9$. 

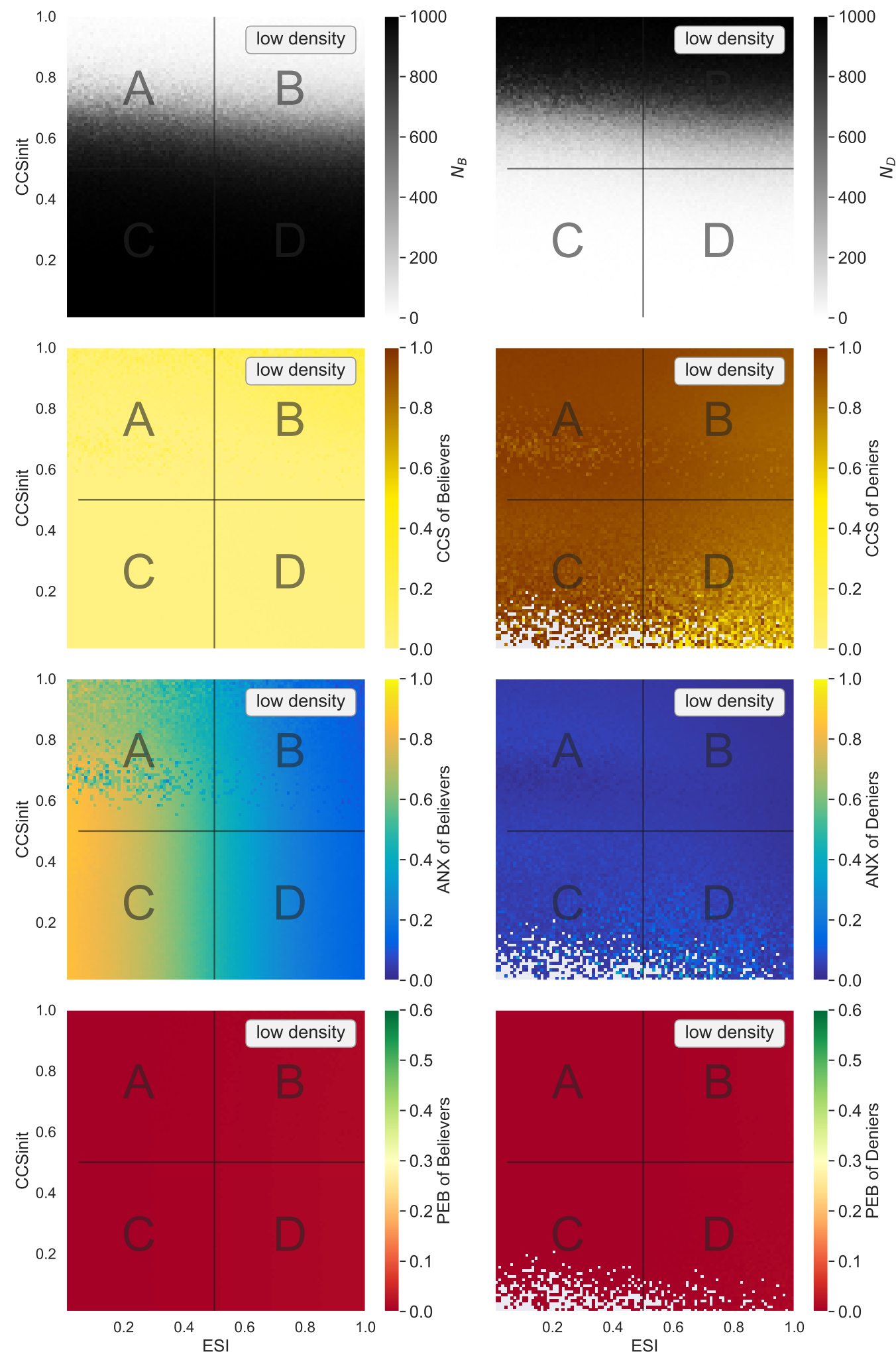

Figure A4. Believers and deniers full parameter plane: Exploration of the initial orientations ESI and CCSinit for the low density case $I I=0.1, I R=0.1$, (left column) believers and (right column) deniers. Parameter $N=1000, I I=0.1, I R=0.1, T_{\text {eq }}=2000-10,000$. 


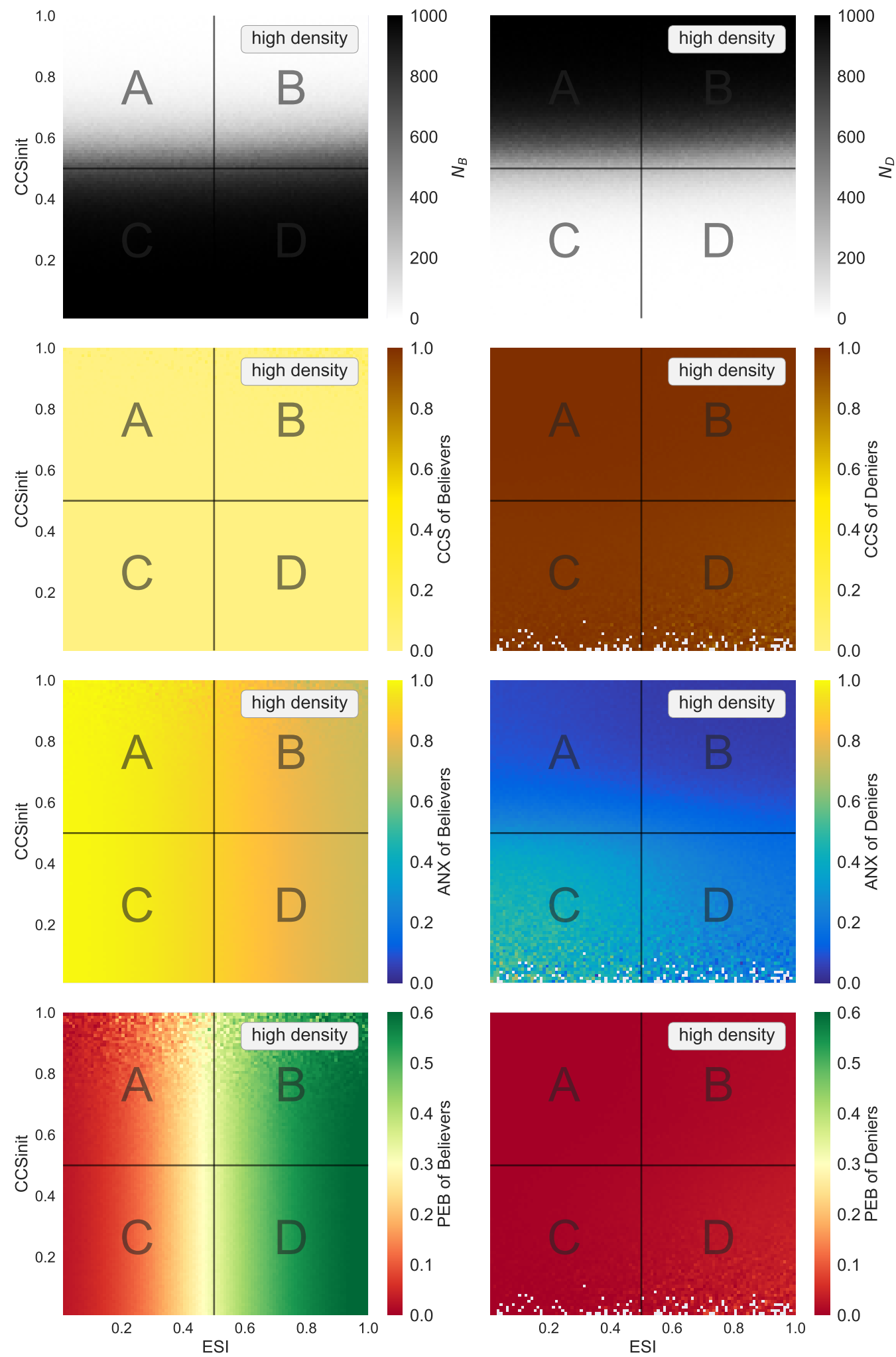

Figure A5. Believers and deniers full parameter plane: Exploration of the initial orientations ESI and CCSinit for the high density case $I I=0.1, I R=0.1$, (left column) believers and (right column) deniers. Parameter $N=1000, I I=0.9, I R=0.9, T_{\text {eq }}=2000-10,000$. 


\section{References}

1. Hedenus, F.; Wirsenius, S.; Johansson, D.J. The importance of reduced meat and dairy consumption for meeting stringent climate change targets. Clim. Chang. 2014, 124, 79-91. [CrossRef]

2. Pardee, V. Up in the Air: How Airplane Carbon Pollution Jeopardizes Global Climate Goals; A Center for Biological Diversity Report; The National Academies of Sciences, Engineering, and Medicine: Washington, DC, USA, 2015.

3. Lenzen, M.; Sun, Y.Y.; Faturay, F.; Ting, Y.P.; Geschke, A.; Malik, A. The carbon footprint of global tourism. Nat. Clim. Chang. 2018, 8, 522. [CrossRef]

4. Emberger, G. Low carbon transport strategy in Europe: A critical review. Int. J. Sustain. Transp. 2017, 11, 31-35. [CrossRef]

5. Magnan, A.; Schipper, E.; Burkett, M.; Bharwani, S.; Burton, I.; Eriksen, S.; Gemenne, F.; Schaar, J.; Ziervogel, G. Addressing the risk of maladaptation to climate change. Wiley Interdiscip. Rev. Clim. Chang. 2016, 7, 646-665. [CrossRef]

6. Moser, S.C.; Ekstrom, J.A. A framework to diagnose barriers to climate change adaptation. Proc. Natl. Acad. Sci. USA 2010, 107, 22026-22031. [CrossRef]

7. Hidalgo, M.C.; Hernández, B.; Lambistos, M.J.; Pisano, I. Evaluating pro-environmental behavior: Differences between low-and high-involvement behaviors. Int. J. Hisp. Psychol. 2011, 4, 45-54.

8. Ramkissoon, H.; Smith, L.D.G.; Weiler, B. Testing the dimensionality of place attachment and its relationships with place satisfaction and pro-environmental behaviours: A structural equation modelling approach. Tour. Manag. 2013, 36, 552-566. [CrossRef]

9. Uhl, I.; Jonas, E.; Klackl, J. When climate change information causes undesirable side effects: The influence of environmental self-identity and biospheric values on threat responses/Cuando la información sobre el cambio climático tiene efectos indeseados: La influencia de la identidad ambiental y de los valores biosféricos en la respuesta ante una amenaza. Psyecology 2016, 7, 307-334.

10. Uhl, I.; Klackl, J.; Hansen, N.; Jonas, E. Undesirable effects of threatening climate change information: A cross-cultural study. Group Process. Intergroup Relat. 2018, 21, 513-529. [CrossRef]

11. Dunlap, R.E. Climate change skepticism and denial: An introduction. Am. Behav. Sci. 2013, 57, $691-698$. [CrossRef]

12. Jonas, E.; McGregor, I.; Klackl, J.; Agroskin, D.; Fritsche, I.; Holbrook, C.; Nash, K.; Proulx, T.; Quirin, M. Threat and defense: From anxiety to approach. In Advances in Experimental Social Psychology; Elsevier: Amsterdam, The Netherlands, 2014; Volume 49, pp. 219-286.

13. Barth, M.; Masson, T.; Fritsche, I.; Ziemer, C.T. Closing ranks: Ingroup norm conformity as a subtle response to threatening climate change. Group Process. Intergroup Relat. 2018, 21, 497-512. [CrossRef]

14. Sparks, P.; Jessop, D.C.; Chapman, J.; Holmes, K. Pro-environmental actions, climate change, and defensiveness: Do self-affirmations make a difference to people's motives and beliefs about making a difference? Br. J. Soc. Psychol. 2010, 49, 553-568. [CrossRef] [PubMed]

15. Carlton, J.S.; Jacobson, S.K. Using expert and non-expert models of climate change to enhance communication. Environ. Commun. 2016, 10, 1-24. [CrossRef]

16. Venhoeven, L.A.; Bolderdijk, J.W.; Steg, L. Explaining the paradox: How pro-environmental behaviour can both thwart and foster well-being. Sustainability 2013, 5, 1372-1386. [CrossRef]

17. Swim, J.K.; Bloodhart, B. Portraying the perils to polar bears: The role of empathic and objective perspective-taking toward animals in climate change communication. Environ. Commun. 2015, 9, 446-468. [CrossRef]

18. McIlroy-Young, B.; Thistlethwaite, J. Canadian Weathercasters' Current and Potential Role as Climate Change Communicators. Environ. Commun. 2019, 13, 834-846. [CrossRef]

19. Jaspal, R.; Nerlich, B.; Cinnirella, M. Human responses to climate change: Social representation, identity and socio-psychological action. Environ. Commun. 2014, 8, 110-130. [CrossRef]

20. Bolderdijk, J.W.; Gorsira, M.; Keizer, K.; Steg, L. Values determine the (in) effectiveness of informational interventions in promoting pro-environmental behavior. PLoS ONE 2013, 8, e83911. [CrossRef]

21. Van der Werff, E.; Steg, L.; Keizer, K. The value of environmental self-identity: The relationship between biospheric values, environmental self-identity and environmental preferences, intentions and behaviour. J. Environ. Psychol. 2013, 34, 55-63. [CrossRef] 
22. Brulle, R.J. From environmental campaigns to advancing the public dialog: Environmental communication for civic engagement. Environ. Commun. 2010, 4, 82-98. [CrossRef]

23. Engels, A.; Hüther, O.; Schäfer, M.; Held, H. Public climate-change skepticism, energy preferences and political participation. Glob. Environ. Chang. 2013, 23, 1018-1027. [CrossRef]

24. Feinberg, M.; Willer, R. Apocalypse soon? Dire messages reduce belief in global warming by contradicting just-world beliefs. Psychol. Sci. 2011, 22, 34-38. [CrossRef] [PubMed]

25. Corner, A.; Whitmarsh, L.; Xenias, D. Uncertainty, scepticism and attitudes towards climate change: Biased assimilation and attitude polarisation. Clim. Chang. 2012, 114, 463-478. [CrossRef]

26. Tobler, C.; Visschers, V.H.; Siegrist, M. Addressing climate change: Determinants of consumers' willingness to act and to support policy measures. J. Environ. Psychol. 2012, 32, 197-207. [CrossRef]

27. Leombruni, L.V. How you talk about climate change matters: A communication network perspective on epistemic skepticism and belief strength. Glob. Environ. Chang. 2015, 35, 148-161. [CrossRef]

28. Gilbert, N. Agent-Based Models; Sage Publications: Thousand Oaks, CA, USA, 2019; Volume 153.

29. Andrei, A.L.; Comer, K.; Koehler, M. An agent-based model of network effects on tax compliance and evasion. J. Econ. Psychol. 2014, 40, 119-133. [CrossRef]

30. Kapeller, M.L.; Füllsack, M.; Jäger, G. Holiday Travel Behaviour and Correlated CO2 Emissions-Modelling Trend and Future Scenarios for Austrian Tourists. Sustainability 2019, 11, 6418. [CrossRef]

31. Natalini, D.; Bravo, G. Encouraging sustainable transport choices in American households: Results from an empirically grounded agent-based model. Sustainability 2014, 6, 50-69. [CrossRef]

32. Ahrweiler, P.; Schilperoord, M.; Pyka, A.; Gilbert, N. Modelling research policy: Ex-ante evaluation of complex policy instruments. J. Artif. Soc. Soc. Simul. 2015, 18, 5. [CrossRef]

33. Soboll, A.; Schmude, J. Simulating tourism water consumption under climate change conditions using agent-based modeling: The example of ski areas. Ann. Assoc. Am. Geogr. 2011, 101, 1049-1066. [CrossRef]

34. Valkering, P.; Tabara, D.; Wallman, P.; Offermans, A. Modelling cultural and behavioural change in water management: An integrated, agent based, gaming approach. Integr. Assess. 2009, 9. Available online: http:/ /116.203.146.222:8080/index.php/iaj/article/viewArticle/285 (accessed on 11 November 2019).

35. Moss, S.; Pahl-Wostl, C.; Downing, T. Agent-based integrated assessment modelling: The example of climate change. Integr. Assess. 2001, 2, 17-30. [CrossRef]

36. Troost, C.; Berger, T. Dealing with uncertainty in agent-based simulation: Farm-level modeling of adaptation to climate change in Southwest Germany. Am. J. Agric. Econ. 2014, 97, 833-854. [CrossRef]

37. Truelove, H.B.; Carrico, A.R.; Thabrew, L. A socio-psychological model for analyzing climate change adaptation: A case study of Sri Lankan paddy farmers. Glob. Environ. Chang. 2015, 31, 85-97. [CrossRef]

38. Downing, T.E.; Moss, S.; Pahl-Wostl, C. Understanding climate policy using participatory agent-based social simulation. In International Workshop on Multi-Agent Systems and Agent-Based Simulation; Springer: Berlin/Heidelberg, Germany, 2000; pp. 198-213.

39. Balbi, S.; Giupponi, C. Agent-based modelling of socio-ecosystems: A methodology for the analysis of adaptation to climate change. Int. J. Agent Technol. Syst. 2010, 2, 17-38. [CrossRef]

40. Gerst, M.D.; Wang, P.; Roventini, A.; Fagiolo, G.; Dosi, G.; Howarth, R.B.; Borsuk, M.E. Agent-based modeling of climate policy: An introduction to the ENGAGE multi-level model framework. Environ. Model. Softw. 2013, 44, 62-75. [CrossRef]

41. Kniveton, D.; Smith, C.; Wood, S. Agent-based model simulations of future changes in migration flows for Burkina Faso. Glob. Environ. Chang. 2011, 21, S34-S40. [CrossRef]

42. Hassani-Mahmooei, B.; Parris, B.W. Climate change and internal migration patterns in Bangladesh: An agent-based model. Environ. Dev. Econ. 2012, 17, 763-780. [CrossRef]

43. Smith, J.W.; Anderson, D.H.; Moore, R.L. Social capital, place meanings, and perceived resilience to climate change. Rural. Sociol. 2012, 77, 380-407. [CrossRef]

44. Grothmann, T.; Patt, A. Adaptive capacity and human cognition: The process of individual adaptation to climate change. Glob. Environ. Chang. 2005, 15, 199-213. [CrossRef]

45. Alvarez-Galvez, J. Network models of minority opinion spreading: Using agent-based modeling to study possible scenarios of social contagion. Soc. Sci. Comput. Rev. 2016, 34, 567-581. [CrossRef]

46. Sopha, B.M.; Klöckner, C.A.; Hertwich, E.G. Adoption and diffusion of heating systems in Norway: Coupling agent-based modeling with empirical research. Environ. Innov. Soc. Transit. 2013, 8, 42-61. [CrossRef] 
47. Kapeller, M.L.; Jäger, G.; Füllsack, M. Homophily in networked agent-based models: A method to generate homophilic attribute distributions to improve upon random distribution approaches. Comput. Soc. Netw. 2019, 6, 9. [CrossRef]

48. Schweitzer, F.; Garcia, D. An agent-based model of collective emotions in online communities. Eur. Phys. J. B 2010, 77, 533-545. [CrossRef]

49. Nardin, L.G.; Andrighetto, G.; Conte, R.; Székely, Á.; Anzola, D.; Elsenbroich, C.; Lotzmann, U.; Neumann, M.; Punzo, V.; Troitzsch, K.G. Simulating protection rackets: A case study of the Sicilian mafia. Auton. Agents -Multi-Agent Syst. 2016, 30, 1117-1147. [CrossRef]

50. Barabási, A.L.; Albert, R. Emergence of scaling in random networks. Science 1999, 286, 509-512. [CrossRef]

51. Stonedahl, F.; Wilensky, U. NetLogo Virus on a Network Model; Center for Connected Learning and Computer-Based Modeling, Northwestern University: Evanston, IL, USA, 2008.

52. Wilensky, U. NetLogo; Center for Connected Learning and Computer-Based Modeling, Northwestern University: Evanston, IL, USA, 2008.

53. Xiang, X.; Kennedy, R.; Madey, G.; Cabaniss, S. Verification and validation of agent-based scientific simulation models. Agent-Dir. Simul. Conf. 2005, 47, 55.

54. Heitzig, J.; Lessmann, K.; Zou, Y. Self-enforcing strategies to deter free-riding in the climate change mitigation game and other repeated public good games. Proc. Natl. Acad. Sci. USA 2011, 108, 15739-15744. [CrossRef]

55. Broersen, J.M.; Dastani, M.; Huang, Z.; Hulstijn, J.; Torre, L. The BOID architecture; Conflicts Between Beliefs, Obligations, Intentions and Desires. In Proceedings of the Fifth International Conference on Autonomous Agents, Montreal, QC, Canada, 28 May-1 June 2001.

56. Chadwick, A.E. Toward a theory of persuasive hope: Effects of cognitive appraisals, hope appeals, and hope in the context of climate change. Health Commun. 2015, 30, 598-611. [CrossRef]

57. Stevenson, K.T.; Peterson, M.N.; Bondell, H.D.; Moore, S.E.; Carrier, S.J. Overcoming skepticism with education: Interacting influences of worldview and climate change knowledge on perceived climate change risk among adolescents. Clim. Chang. 2014, 126, 293-304. [CrossRef]

58. Zannakis, M.; Molander, S.; Johansson, L.O. On the Relationship between Pro-Environmental Behavior, Experienced Monetary Costs, and Psychological Gains. Sustainability 2019, 11, 5467. [CrossRef]

59. Wahlström, M.; Sommer, M.; Kocyba, P.; de Vydt, M.; De Moor, J.; Davies, S.; Wouters, R.; Wennerhag, M.; van Stekelenburg, J.; Uba, K.; et al. Protest for a Future: Composition, Mobilization and Motives of The Participants in Fridays for Future Climate Protests on 15 March, 2019 in 13 European Cities. 2019. Available online: http:/ / eprints.keele.ac.uk/6571/ (accessed on 11 November 2019).

60. Kaiser, F.G.; Brügger, A.; Hartig, T.; Bogner, F.X.; Gutscher, H. Appreciation of nature and appreciation of environmental protection: How stable are these attitudes and which comes first? Rev. Eur. Psychol. AppliquÉE/European Rev. Appl. Psychol. 2014, 64, 269-277. [CrossRef]

61. Ireland, L. Educating for the 21st Century: Advancing an Ecologically Sustainable Society. Ph.D. Thesis, University of Stirling, Stirling, Scotland, 2007.

62. Prévot, A.C.; Clayton, S.; Mathevet, R. The relationship of childhood upbringing and university degree program to environmental identity: Experience in nature matters. Environ. Educ. Res. 2018, 24, $263-279$. [CrossRef]

63. Standish, R.J.; Hobbs, R.J.; Miller, J.R. Improving city life: Options for ecological restoration in urban landscapes and how these might influence interactions between people and nature. Landsc. Ecol. 2013, 28, 1213-1221. [CrossRef]

64. Di Fabio, A. The psychology of sustainability and sustainable development for well-being in organizations. Front. Psychol. 2017, 8, 1534. [CrossRef] [PubMed]

(C) 2020 by the authors. Licensee MDPI, Basel, Switzerland. This article is an open access article distributed under the terms and conditions of the Creative Commons Attribution (CC BY) license (http:/ / creativecommons.org/licenses/by/4.0/). 